Schweizerisches Jahrbuch für Entwicklungspolitik

27-2 | 2008

Migration und Entwicklung : Eine Zweckallianz

\title{
Historische und soziologische Übersicht über die Migration in der Schweiz
}

\section{Gianni D'Amato}

\section{(2) OpenEdition \\ 1 Journals}

Electronic version

URL: https://journals.openedition.org/sjep/340

DOI: $10.4000 /$ sjep.340

ISSN: 1663-9677

Publisher

Institut de hautes études internationales et du développement

Printed version

Date of publication: 1 décembre 2008

Number of pages: 177-195

ISBN: 978-2-940415-08-3

ISSN: 1660-5926

\section{Electronic reference}

Gianni D'Amato, „Historische und soziologische Übersicht über die Migration in der Schweiz",

Schweizerisches Jahrbuch für Entwicklungspolitik [Online], 27-2 | 2008, Online erschienen am: 31 mai 2010, abgerufen am 28 juin 2022. URL: http://journals.openedition.org/sjep/340 ; DOI: https://doi.org/ $10.4000 /$ sjep.340 


\title{
Historische und soziologische Übersicht über die Migration in der Schweiz
}

\author{
Gianni D'Amato*
}

\section{Der „verspätete“ Blick von Migrationsforschung auf die Realität}

Eine Inventarisierung des Migrationsphänomens in der Schweiz, so unvollständig deren Darstellung in diesem Beitrag auch sein mag, bietet letztlich die Chance, festgefahrene Paradigmen der sozialwissenschaftlichen Migrationsforschung erneut kritisch zu reflektieren und eine neue Übersicht über die unter hohem Handlungszwang stehenden politischen Bereich zu gewinnen. Der Druck im universitären Forschungsmanagement hat ja bekanntlich dazu geführt, dass spezialisierte akademische Institutionen häufig den Konjunkturen von Immigrationsdebatten und -politiken folgen, anstatt längerfristig Forschungsressourcen bereitzustellen, die es ermöglichen, die Einwanderung interdisziplinär und kontinuierlich als festen Bestandteil moderner Gesellschaften zu untersuchen.

Doch trotz der tagespolitischen und thematischen Brisanz hat es sich die Migrationsforschung bislang entgehen lassen, die Komplexität der Materie und die widersprüchlichen Interessen der Akteure - der Migranten und Migrantinnen wie auch der Ein- und Auswanderungsländer - theoretisch so zu erfassen, dass die Resultate dieser Analyse auch für eine inklusive Gestaltung der Politik hätten nutzbar gemacht werden können. Dieser Unterlassungssünde seitens der Wissenschaft entspricht auch das Versagen der Politik, die es in den letzten dreissig bis vierzig Jahren in vielen europäischen Ländern versäumt hat, den Wandel von der „Gastarbeiterbevölkerung“ zur Einwanderungsminorität rechtzeitig zur Kenntnis zu nehmen und auf Basis der neuen Umstände zukunftsfähige bevölkerungspolitische Konzepte zu entwerfen ${ }^{1}$. Die nun folgende Diskussion möchte einen knappen kritischen Überblick über die wichtigsten Dimensionen des Migrationsgeschehens in der Schweiz vermitteln und Anhaltspunkte dafür liefern, wie Migration und Entwicklung in einem Kontext globaler gesellschaftlicher Transformationen verstanden werden kann.

\section{Einwanderungsland Schweiz?}

Die Schweiz war seit Jahrhunderten stets bekannt für ihre Neutralität, ihre wirtschaftliche und gesellschaftliche Stabilität, ihre sprachliche und kulturelle Vielfalt und ihre Kombination aus direkter Demokratie und Föderalismus. Die daraus resultierende Reputation als Hort der Freiheit wirkte seit der Neuzeit

* Professor und Direktor, Schweizerisches Forum für Migrations- und Bevölkerungsstudien, Universität Neuenburg.

1 Eine erste Kritik in diesem Sinne findet sich bei Kingsley Davis, ,Social Science Approaches to International Migration“, Population and Development Review, Bd. 14, Nr. 4, 1988, S. 245. 
anziehend auf verschiedene Menschen in Europa, die Zuflucht vor Verfolgung, aber auch eine neue wirtschaftliche und geistig-politische Heimstätte suchten. Die Hugenotten und andere protestantische Flüchtlinge gehörten zu den Ersten, die im sechzehnten und siebzehnten Jahrhundert im Gefolge der Bartholomäusnacht und des Dreissigjährigen Krieges Schutz fanden. Im 19. Jahrhundert profitierten verschiedene europäische Exilanten im gleichen Masse vom Triumph des schweizerischen Liberalismus, und fanden hauptsächlich nach dem Scheitern ihrer Bewegungen im März 1848 Unterschlupf in der Eidgenossenschaft ${ }^{2}$.

Der Wandel der Schweiz zu einem Einwanderungsland moderner Prägung setzte erst nach der beschleunigten Industrialisierung in der zweiten Hälfte des 19. Jahrhunderts richtig ein. Anders als das von der romantischen Literatur gepflegte Bild eines rein ländlich-alpinen Raumes gehörte die Schweiz schon damals zur europäischen Vorhut in verschiedenen Branchen, zunächst der Textil-, später auch der mechanischen und chemischen Industrie. Diese Vorrangstellung war gekennzeichnet durch einen hohen Bedarf an Investitionen in der Industrie, bei der Infrastruktur aber auch in der universitären Wissensproduktion und -vermittlung. Gleichzeitig schrumpfte der Landwirtschaftssektor, und viele dort tätige Personen wanderten entweder in die Industrie ab oder verliessen die Schweiz, um in Übersee eine neue Existenz aufzubauen ${ }^{3}$. Bereits seit Mitte des 19. Jahrhunderts wanderten insbesondere deutsche Akademiker, Selbständige und Handwerker ein, aber auch Italiener, die in der Wissenschaft, der Industrie, dem Baugewerbe und dem Infrastrukturbau eine Beschäftigung fanden ${ }^{4}$. Insbesondere in der Periode zwischen 1888 und 1914 stieg deren Zahl rasant an. Zwischen 1888 und 1919 verdoppelte sich die Zahl der Deutschen von 112000 auf 220000, während die italienische Gemeinde zwischen 1900 und 1910 von 117000 auf 203000 wuchs $^{5}$.

In der Phase vor dem Ersten Weltkrieg oblag die Verantwortung für die Migrationspolitik den Kantonen, deren Gesetze allerdings den Bedingungen bilateraler Vereinbarungen zu entsprechen hatten, mit denen die Schweiz und andere Staaten die Zuwanderung regulierten. Wie andere Vereinbarungen jener Zeit betreffend der Freizügigkeit standen diese der Einwanderung nicht ablehnend gegenüber, da sie auch den eigenen Bürgern eine Auswanderung sichern wollten. Allerdings wurde nach einer ersten Kampagne gegen die Anwesenheit von Fremden gegen Ende des Ersten Weltkriegs schon 1925 von der Bevölkerung

2 Thomas Busset „La politique du refuge en Suisse 1820-1870. Réalité et mythe“, Zeitschrift des Schweizerischen Bundesarchivs, Nr. 25, 1999, S. 34.

3 Dieter Freiburghaus und Brigitte Guggisberg, „Die schweizerische Ausländerpolitik seit 1850: eine Analyse auf dem Hintergrund politisch-ökonomischer Paradigmen“, in Thomas Geiser, Hans Schmid und Emil Walter-Busch (Hg.), Arbeit in der Schweiz des 20. Jahrhunderts: wirtschaftliche, rechtliche und soziale Perspektiven, Bern, P. Haupt, 1998, S. 137-185.

4 Die Heterogenität der deutschen Einwanderung im 19. Jahrhundert, aber auch deren Rolle in der Entwicklung des wirtschaftlichen, kulturellen und politischen Lebens der Schweiz vor dem Ersten Weltkrieg, zeichnet eine Studie von Klaus Urner im Detail nach (siehe Klaus Urner, Die Deutschen in der Schweiz: von den Anfängen der Kolonienbildung bis zum Ausbruch des Ersten Weltkrieges, Frauenfeld, Huber, 1976). Zu den Italienern in der Schweiz siehe die Schriften von Vuilleumier (Marc Vuilleumier, „L'emigrazione italiana in Svizzera e gli avvenimenti del 1898“, in AA. VV., Anna Kuliscioff e l'età del riformismo: atti del convegno di Milano, dicembre 1976, Roma, Mondo operaio, 1978, S. 85-103 ; Marc Vuilleumier, „Le mouvement ouvrier et les travailleurs italiens en Suisse jusqu'à la Première Guerre mondiale“, Revue syndicale suisse, Bd. 82, Nr. 4, 1990, S. 102-116).

5 Siehe Madelyn Holmes, Forgotten Migrants: Foreign Workers in Switzerland before World War I, Rutherford, Fairleigh Dickinson University Press, 1998, S. 15. 
ein Verfassungszusatz angenommen, der dem Bundesrat die Macht verlieh, die Niederlassung von Ausländerinnen und Ausländern auf Bundesebene zu regeln. Ausserdem erhielt er den Verfassungsrahmen für den Aufbau der Fremdenpolizei und die Formulierung des 1931 vorgeschlagenen Bundesgesetzes über den Aufenthalt und die Niederlassung von Ausländern (ANAG). Von da an musste die Zuwanderung sowohl mit den moralischen und wirtschaftlichen Interessen der Schweiz im Einklang stehen als auch den Grad der „Überfremdung“ berücksichtigen. Der politische Konsens in Fragen der kulturellen und demographischen „Fremdenabwehr" hat bis zum 2008 in Kraft getretenen neuen Ausländergesetz $(\mathrm{AuG})$ verhindert, dass über die letzten Jahrzehnte eine kohärent auf Integration ausgerichtete Einwanderungspolitik hätte betrieben werden können. Von Arbeitsmigrantinnen und -migranten wurde implizit erwartet, dass sie das Land nach getaner Arbeit wieder verliessen.

\section{Die Arbeitsmigration nach dem Zweiten Weltkrieg}

Nach dem Zweiten Weltkrieg hat das Bedürfnis westlicher Staaten, sich ökonomisch innerhalb der von den Bretton-Woods-Institutionen getragenen Marktordnung $\mathrm{zu}$ stabilisieren, $\mathrm{zu}$ einer erneuten Förderung der Auswanderung geführt. In Erwartung einer einsetzenden Konjunktur unterzeichnete die Schweiz bereits 1948 mit Italien ein Rekrutierungsabkommen. Die meisten der italienischen Arbeitskräfte wurden in der Folge im Infrastruktur- und Bausektor eingesetzt, aber auch in der Maschinen- und Textilindustrie.

Mit der Verrechtlichung der Migrantenfrage durch das ANAG standen nun Verfahren zur Verfügung, die den Zugang zum Schweizer Arbeitsmarkt detailliert regelten. Das flexible System der Niederlassung sah unterschiedliche Bewilligungen vor, wie etwa eine Saisonnierbewilligung (Ausweis A), Jahresaufenthaltsbewilligung (Ausweis B), Niederlassungsbewilligung (Ausweis C), Kurzaufenthaltsbewilligung (Ausweis L) und eine Bewilligungen für Asylbewerber (Ausweis N), auf deren Lage wir später gesondert zu sprechen kommen. Migrationspolitik kann in diesem Sinn als gezielter Versuch verstanden werden, mit Hilfe staatlicher Instrumente Einfluss auf die Migationsentwicklung zu nehmen. Besonders die Zulassungspolitik stand permanent unter dem Kreuzfeuer der Kritik. So wurde gleich nach 1945, um sicherzustellen, dass die Migrantinnen und Migranten sich nicht auf Dauer in der Schweiz niederliessen, die Dauer des Aufenthalts, die Anrecht auf eine längerfristige Niederlassung gab, von fünf auf zehn Jahre verlängert. Auch wurden die Bedingungen für die Familienzusammenführung restriktiver ausgelegt. Die Politik setzte auf die Rotation ausländischer Arbeitskräfte, also auf eine kontinuierliche Ersetzung der Migranten durch neue, anzulernende Arbeiter, damit diese nicht sesshaft werden konnten und zur „Überfremdung" des Landes beitrugen. Deshalb erhielten die Arbeiterinnen und Arbeiter zu Beginn ihres Einsatzes die Arbeitsbewilligung nur für die Dauer eines Jahres; diese konnte allerdings verlängert werden, was auch häufig geschah.

Seit der Wiederaufnahme der Rekrutierungspolitik stieg die Zahl der Immigranten, die sich endgültig in der Schweiz niederliessen, in zehn Jahren von 271000

6 Hans Mahnig und Etienne Piguet, „Die Immigrationspolitik der Schweiz von 1948 bis 1998 : Entwicklung und Auswirkungen“, in Hans-Rudolf Wicker, Rosita Fibbi und Werner Haug (Hg.), Migration und 
(1950) auf 476000 (1960) an, bis sie 19701080000 betrug $^{6}$. Kamen diese Arbeitskräfte zunächst mehrheitlich aus Italien, diversifizierten sich die Herkunftsregionen ab den 1960er Jahren: 1970 stammten mehr als die Hälfte der Ausländerinnen und Ausländer immer noch aus Italien, aber 20 Prozent kamen derweil aus Deutschland, Frankreich und Österreich, 10 Prozent aus Spanien und 4 Prozent aus Jugoslawien, Portugal und der Türkei.

Mit dem Andauern der wirtschaftlichen Hochkonjunktur wurde in den 1960er Jahren dem Rotationsmodell sowohl von der Wirtschaft als auch von der Politik immer weniger Beachtung geschenkt. Ausserdem verbesserten sich die Aufenthaltsbedingungen der Zuwanderer in der Schweiz, nicht zuletzt aufgrund der in ganz Europa stetig wachsenden Nachfrage nach Arbeitskräften und den sozialpolitischen Pressionen der Herkunftsländer. Unter diesen veränderten Rahmenbedingungen musste die schweizerische Wirtschaftsdiplomatie Konzessionen zugestehen ${ }^{7}$. Im Gefolge des zweiten bilateralen Rekrutierungsabkommens mit Italien 1964 versuchten die Schweizer Behörden ihr Rotationssystem mit einem integrationsorientierten Modell zu ersetzen. Auch die Vertreter der Migranten hatten bereits in den 1970er Jahren die Integration der ausländischen Arbeitskräfte als gleichberechtigte Anerkennung in Gesellschaft, Wirtschaft und Politik thematisiert. Die verstärkte Promotion eigener Interessen in den Gewerkschaften, die Familienzusammenführung, die Aufhebung von schulischen Sonderklassen und anderen Formen schulischer Ungleichbehandlung waren ihre wichtigsten Forderungen. Diese seitens der Migrantenorganisationen öffentlich formulierten Ansprüche führten indes auch zu einer Revitalisierung der „Überfremdungsbewegung“, welche ihrerseits die Reduzierung der Ausländerbestände und die Verweigerung einer Expansion sozialer Rechte verlangte. Die Gewerkschaften waren in der Frage der Integration gespalten: Die Einsicht in die Notwendigkeit, ausländische Arbeitskräfte in den eigenen Reihen aufzunehmen, konkurrierte mit der Vorstellung etlicher Funktionäre, die „Fremdarbeiter“ seien nichts anderes als Konkurrenten auf dem Arbeitsmarkt ${ }^{8}$.

Nach dem Ölschock von 1973 und dem darauf folgenden Konjunkturabschwung mussten viele ausländische Arbeitskräfte das Land verlassen, da etliche unter ihnen dazumal über keine Arbeitslosenversicherung verfügten. Dieser Umstand erlaubte der Schweiz, die Arbeitslosigkeit durch die Limitierung der Aufenthaltserlaubnisse in die Herkunftsländer zu exportieren. Der Anteil der ausländischen Bevölkerung nahm von 17.2 Prozent auf 14.8 Prozent ab. Mit der Erholung der wirtschaftlichen Situation Ende der 1970er Jahre kamen nun die neuen „Gastarbeiter" nicht mehr nur aus Italien, sondern vermehrt auch aus Spanien, Portugal und der Türkei. Der Anteil der Zuwanderer stieg wieder von 14.8 Prozent (1980) auf 16.4 Prozent (1990) und 19.5 Prozent (2000) (siehe Grafik 1).

die Schweiz: Ergebnisse des Nationalen Forschungsprogramms „Migration und interkulturelle Beziehungen", Zürich, Seismo, 2003, S. 65-108.

7 Mauro Cerutti, „L'immigration italienne en Suisse dans le contexte de la Guerre froide“, in Jean Batou, Mauro Cerutti und Charles Heimberg (Hg.), Pour une histoire des gens sans histoire: ouvriers, exclus et rebelles en Suisse: XIXe-XXe siècles, Lausanne, Editions d'En Bas, 1995, S. 213-231.

8 Erwin Carigiet, Ueli Mäder und Jean-Michel Bonvin (Hg.), Wörterbuch der Sozialpolitik, Zürich, Rotpunktverlag, 2003, S. 206. 
Grafik 1: Ausländeranteil an der gesamten ständigen Wohnbevölkerung, 1860-2007 (in Prozent)

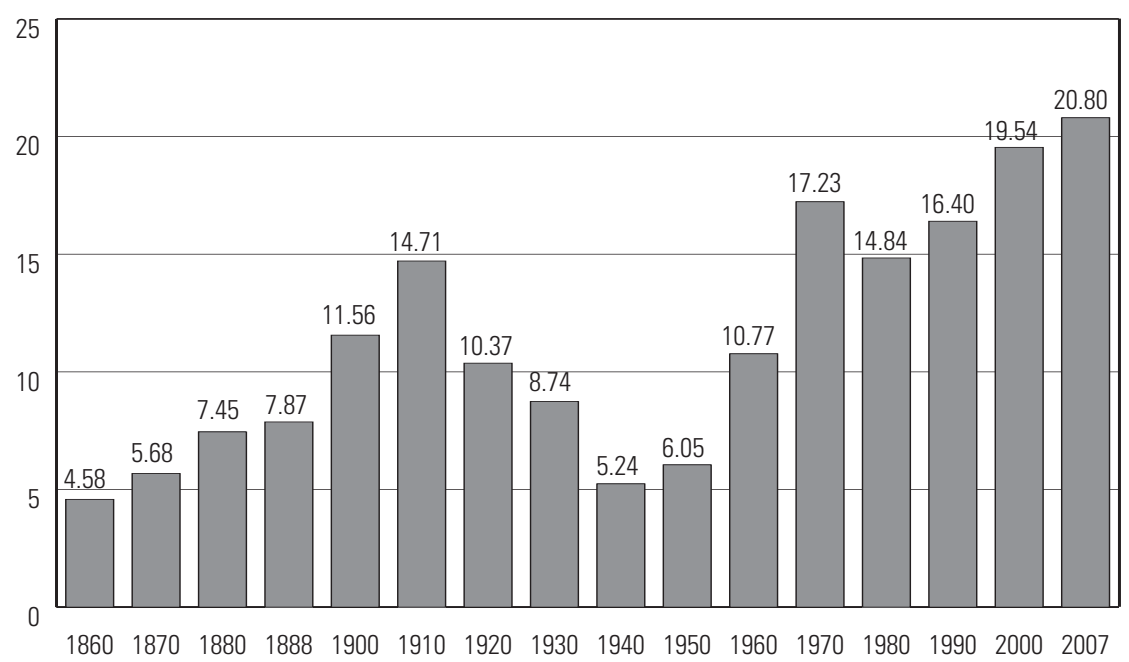

Quellen: Bundesamt für Statistik (BFS) (Hrsg.), Statistisches Jahrbuch der Schweiz 2008, Neuenburg, BFS. Bundesamt für Migration (BFM), „Ausländerstatistik“, 2007.

Die Wirtschaftskrise der 1990er Jahre traf erneut die unqualifizierten und nun in die Jahre gekommenen ausländischen Arbeitskräfte, in deren Reihen die Arbeitslosigkeit hoch war und die Mühe hatten, aufgrund ihrer nicht mehr gebrauchten Qualifikationen den Wiedereinstieg in die Arbeitswelt zu finden. Diese Situation führte zu einer strukturellen Arbeitslosigkeit, die die Schweiz in diesem Masse seit dem Zweiten Weltkrieg nicht mehr erlebt hat. Verschiedene Städte, die einen grossen Teil der sozialen Folgekosten dieser Krise tragen mussten, verlangten vom Bund ein stärkeres Engagement und hatten damit Erfolg. Mit dem 1999 in Kraft getretenen Integrationsartikel beteiligte sich der Bund erstmals finanziell an der kommunalen Integrationsförderung ${ }^{9}$. Die Bedeutung von Integration erhielt dabei eine neue Wendung. Nebst der Frage gegenseitige Anerkennung ging es erstmals auch um die Schaffung von Kompetenzen in der Migrations- und in der Schweizer Bevölkerung ${ }^{10}$. Alle Bevölkerungsteile sollten sich in Zukunft aufeinander beziehen können. Sprachkompetenz, Austausch, Vermeidung von Zugangsbarrieren in Erziehung, Gesundheit und bei den Behörden waren die wichtigsten Ziele.

Das neue Ausländergesetz (AuG), das 2008 in Kraft getreten ist und das ANAG von 1931 ersetzt hat, enthält dementsprechend erstmals ein im Gesetz verankertes Kapitel zur Integration, dass ebenfalls als Forderung an die Ausländer gelesen werden kann, sich in die Gesellschaft einzugliedern oder zu gehen. Das neue AuG stellt nun auch die Interessen der gesamten Wirtschaft voran, nicht bloss jene von spezifischen Branchen, und zielt insbesondere auf die Einwanderung von nichteuropäischen hochqualifizierten Arbeitskräften oder jene von

9 Gianni D’Amato und Brigitta Gerber (Hg.), Herausforderung Integration: städtische Migrationspolitik in der Schweiz und in Europa, Zürich, Seismo, 2005.

10 Erwin Carigiet et al., op. cit. 
Bürgerinnen und Bürgern der EFTA und EU, deren Zulassung über die 2002 in Kraft getretenen bilateralen Verträge mit der EU geregelt ist. Ausländer mit einer Aufenthaltsgenehmigung verfügen mit dem neuen Ausländergesetz über bessere Freizügigkeitsbedingungen auf dem inländischen Arbeitsmarkt und dürfen beispielsweise sofort ihre Familien nachziehen lassen.

Ein Blick in die Statistiken offenbart, wie rasch Gesetzesänderungen den gewandelten Bedürfnissen der Wirtschaft folgen und wie duktil Migrationsströme auf gewandelte Rahmenbedingungen reagieren. Waren 1970 in der Hochkonjunktur noch rund 300000 ausländische Arbeiter in der Industrie beschäftigt (46\% aller arbeitstätigen Ausländer), sind es 2005 nur noch 169000 $(20,4 \%)^{11}$. Stetig abgenommen hat seither auch der Beschäftigungsanteil im Baugewerbe, während jener in der Hotellerie zwischen 1970 und 2005 leicht zugenommen hat. Die reduzierte Bedeutung dieser drei Branchen, in denen Ausländer seit dem Zweiten Weltkrieg traditionell beschäftig waren, ist dem steten Aufstieg der Dienstleistungsökonomie zuzuschreiben. Waren im Kredit-, Immobilien und Versicherungsgewerbe 1970 erst 18000 Personen ausländischer Nationalität tätig (2,7\% der insgesamt beschäftigten ausländischen Arbeitskräfte), so waren es 2005133000 Personen (16,1\%). Der Strukturwandel der Wirtschaft äussert sich auch in der Diversifizierung der Herkunftsgebiete: Seit der Jahrtausendwende und mit Inkraftsetzung der Bilateralen Verträge nimmt die Einwanderung insbesondere von Deutschen und Portugiesen konstant zu, während jene der Angehörigen der klassischen Rekrutierungsländer (Italien, Spanien, ehemaliges Jugoslawien) beständig abnimmt. Dies hat nicht zuletzt mit der Rückkehr vieler älterer Migrantinnen und Migranten in ihre Herkunftsländer zu tun, aber auch mit dem Einbürgerungsverhalten der „alten“ Einwanderungsgruppen in der Schweiz. Die Deutschen entsprechen in ihrem Profil grösstenteils der von der Wirtschaft für die Dienstleistungsökonomie gewünschten Qualifikationen, die Portugiesen hingegen sind insbesondere im Niedriglohnsektor tätig, für den es nach wie vor grossen Bedarf gibt ${ }^{12}$. Die Ausländerinnen und Ausländer, die zu Erwerbszwecken in die Schweiz kommen, machen 2007 bereits 44.4 Prozent der jährlichen Einwanderung aus (2004: $35 \%$ ). Viele ausländische Menschen kommen ausserdem über den Familiennachzug in die Schweiz (32.3\%, 2004: 40.3\%). Ein weiterer grosser Anteil wandert zum Zwecke der Aus- und Weiterbildung ein (10.5\%) (siehe Grafik 2).

Wenn von Migrantinnen und Migranten in der Schweiz die Rede ist, sind meist auch die Angehörigen der zweiten Generation gemeint, also jene Jungen, die entweder in der Schweiz geboren oder grösstenteils aufgewachsen sind, ohne indes je selbst gewandert zu sein. Während ältere Studien deren Lebenssituation meist als Ergebnis anomiegenerierender Bedingungen darstellten, haben neuere Forschungen dieses Bild korrigiert ${ }^{13}$. Werden die eingebürgerten jungen Leute mitgerechnet, erscheinen Jugendliche mit Migrationshintergrund im Vergleich zu gleichaltrigen Schweizern mit ähnlicher sozialer Herkunft als „Overperfor-

11 Selbstverständlich waren auch Schweizer im verarbeitenden Gewerbe beschäftigt. Die Zahlen hierfür : 741775 (31,8\%) für 1970, 476000 für 2005 (15,1\%). Angaben aus Etienne Piguet, Einwanderungsland Schweiz: fünf Jahrzehnte halb geöffnete Grenzen, Bern, Haupt Verlag, 2006.

12 Siehe Philippe Wanner, Migration und Integration: Ausländerinnen und Ausländer in der Schweiz, Neuenburg, Bundesamt für Statistik, 2004, S. 61.

13 Claudio Bolzman, Rosita Fibbi und Marie Vial, Secondas - secondos : le processus d'intégration des jeunes adultes issus de la migration espagnole et italienne en Suisse, Zurich, Seismo, 2003. 
mer", insbesondere was die soziale Mobilität angeht. Auch wenn sie strukturell gut integriert sind, ist auffällig, wie sie die Welt ihrer Herkunftsfamilie und jener ihrer schweizerischen Umgebung kulturell in der Balance halten. Dieser Befund einer „segmentierten Assimilation“ (Alejandro Portes) wurde von einer Studie des Bundesamtes für Statistik bestätigt und präzisiert: Das vergleichsweise gute Abschneiden der zweiten Generation kann bei allen Jugendlichen mit Migrationshintergrund festgestellt werden, die in der Schweiz geboren wurden, unabhängig vom Zeitpunkt der Einwanderung und ihrem legalen Status (Ausländer, Schweizer). Allerdings ist der Besitz der Schweizer Staatsbürgerschaft keine Hilfe für schlecht qualifizierte Jugendliche mit Migrationshintergrund, insbesondere wenn sie im Ausland geboren sind ${ }^{14}$.

\section{Grafik 2: In die Schweiz eingereiste Ausländer nach Einwanderungsrund, 2007 (in Prozent)}

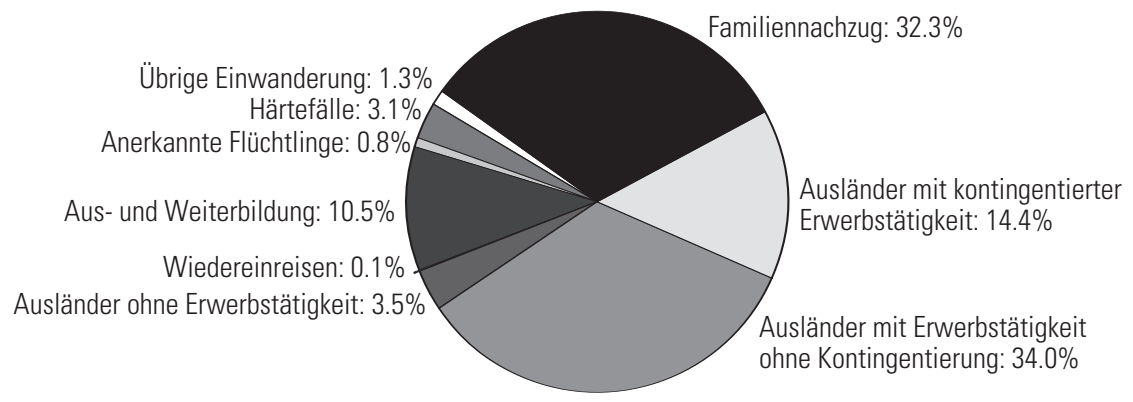

Quelle: Bundesamt für Migration (BFM), „Ausländerstatistik“, 2007.

Der Wandel der gesetzlichen Rahmenbedingungen hat üblicherweise einen Einfluss auf die Aufenthaltstitel der ausländischen Wohnbevölkerung in der Schweiz. So verfügen Angehörige der italienischen, spanischen und portugiesischen Community in der Schweiz zu einem überwiegend grossen Teil über die Niederlassungsbewilligung (Ausweis C; Italiener: 94\%, Spanier: 93\%, Portugiesen : 83\%), während weniger als 40 Prozent der Afrikaner, Asiaten und Amerikaner über diesen Status verfügen. Ausserdem haben 13 Prozent der Afrikaner und Asiaten einen Asylbewerberstatus (Ausweis N), 11 Prozent sind vorläufig Aufgenommene (Ausweis F) und 7 Prozent verfügen lediglich über eine Kurzaufenthaltsbewilligung (Ausweis L). Zwei Drittel der Franzosen und Deutschen haben einen Ausweis $C$, ein Drittel unter ihnen verfügt aber entweder über eine Jahresaufenthaltsbewilligung (Ausweis B) oder die Berechtigung zum Kurzaufenthalt (siehe Grafik 3). Daraus lässt sich schliessen, dass Herkunft und Einwanderungspfad eng verknüpft sind mit der Aufenthaltssicherheit in der Schweiz. Mit den Bilateralen Abkommen, wie erwähnt 2002 in Kraft getreten, haben sich ausserdem die Aufenthaltsbedingungen der Angehörigen der Europäischen Union schlagartig verbessert. Etliche Personen, die vor dem Stichdatum ohne Aufenthaltsberechtigung in der Schweiz lebten (u.a. Portugiesen), konnten in der Folge ihren Aufenthalt regularisieren. Nichteuropäer, insbesondere jene unter dem Titel eines temporären Aufenthalts (im Jahr 2007: 29298 Personen),

14 Rosita Fibbi, Mathias Lerch und Philippe Wanner, L'intégration des populations issues de l'immigration en Suisse: personnes naturalisés et deuxième génération, Neuchâtel, Office fédéral de la statistique, 2005, S. 57. 
verfügen nur über beschränkte Rechte in Bezug auf Freizügigkeit, Integration und Familiennachzug. Dementsprechend unsicher gestalten sich deren Perspektiven in der Schweiz. Dies trifft auch auf die 12243 Asylbewerber (Jahr 2007) zu, die während ihres Anerkennungsverfahrens nur einen begrenzten Zugang zum Arbeitsmarkt haben.

\section{Grafik 3: Bestand der ständigen ausländischen Bevölkerung, 1950-2007 (in Tausenden)}

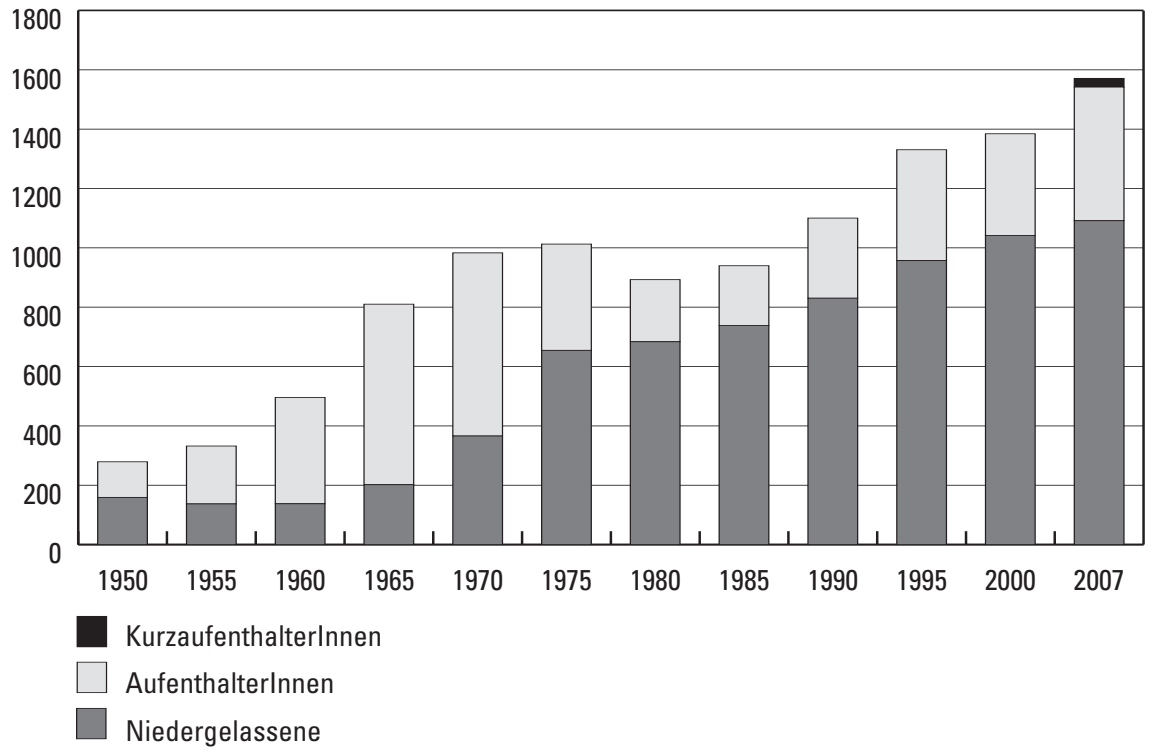

Quelle: Bundesamt für Migration (BFM), „Ausländerstatistik“, 2007.

Die erfolgreiche Dualisierung der Zulassungs- und Arbeitsmarktpolitik im Gefolge der Unterzeichnung des Freizügigkeitsabkommen mit der Europäischen Union hat die Schweizer Migrationspolitik nachhaltig geprägt. Nicht nur gehören die Kontingente und der Inländervorrang, jene Markenzeichen der korporatistischen Zuwanderungspolitik zum Schutze branchenspezifischer und regionaler Interessen, der Vergangenheit an. Auch die sektorielle Herangehensweise an migrationspolitische Fragestellungen musste in der Folge relativiert, wenn nicht aufgegeben werden. Denn die differenzierte Zulassungspolitik hatte einige nichtintendierte Wirkungen zur Folge, sowohl auf der Ebene künftiger Migrationsströme als auch auf deren politisch-administrative Bewältigung. Konzipiert wurde diese Einwanderungspolitik von der Verwaltung in den 1990er Jahren, um die „Angst vor der Überfremdung“ zu überwinden und die Annäherung der Schweiz an Europa zu ermöglichen, da man davon ausging, dass sowohl europäische Arbeitskräfte als auch hochqualifizierte Nichteuropäer weniger Ressentiments in der Bevölkerung erzeugen würden.

Konsequenterweise führte Anfang der 1990er Jahre die Relegation traditioneller Rekrutierungsgebiete wie das ehemalige Jugoslawien vom zweiten in den dritten „Kreis“, eine Antwort des Bundesrates auf die menschenrechtlich schwieriger werdende Lage kurz vor Beginn der Sezessionskriege, zu einer Schliessung saisonaler Rekrutierungskanäle. Der Wille, Europa eine Öffnung zu signalisieren und gleichzeitig die Rekrutierung von qualifizierten Arbeitskräften aus der 
EU oder den EFTA-Staaten zu fördern, führte freilich ungewollterweise zu einem Anstieg der Asylgesuche, obwohl schon damals ein Ausweichen auf den Asylkanal prognostiziert wurde, falls die jugoslawischen Arbeitnehmerinnen und Arbeitnehmer aus den offiziellen Rekrutierungsländern ausgeschlossen würden.

Doch die Verwaltung lernte aus gemachten Fehlern. Angesichts der komplexen Thematik gewannen die Argumente der Befürworter einer ganzheitlichen Migrationspolitik um den damaligen Direktor des Bundesamtes für Flüchtlinge, Peter Arbenz, bald an Gewicht. Im Parlament fand der Vorschlag breite Zustimmung, wonach der Bundesrat ein Migrationsgesetz auszuarbeiten habe, das eine ausgeglichene Auswanderungsbilanz nach Kriterien gewährleisten sollte, die ebenso humanitären Gesichtspunkten Rechnung trugen wie wirtschaftlichen und politischen ${ }^{15}$. Im Raum stand auch die Forderung der staatspolitischen Kommission, ein neues Migrationsgesetz solle die Zielsetzungen und Grenzen der Einwanderung festhalten, eine umfassende Integrationspolitik festlegen, Grundlagen für die Förderung der Rückkehr ins Heimatland schaffen und zudem dazu beitragen, die Ursachen der Migration zu beseitigen. Damit war der Grundstein für eine migrationspolitische Gesamtstrategie gelegt, die über geeignete Instrumente der Politikkoordination die verschiedenen Bereiche der Migrations-, Asyl- und Entwicklungspolitik führen will. Diese gesamtheitliche Sicht auf die verschiedenen Aspekte der Migration findet ihre Spiegelung in der Schaffung interdepartementaler Arbeitsgruppen (siehe IDAG Migration), der Unterstützung internationaler Initiativen von Migrationspartnerschaften seitens der Schweiz (Berne Initiative, Global Commission on International Migration, UNHigh Level Dialogue on International Migration and Development, Global Forum on International Migration and Development) und selbst im neuen Ausländer- (AuG) und Asylgesetz (AsylG), welche beide Elemente der Migrationspartnerschaft aufgenommen haben (siehe Art. $100 \mathrm{AuG}$ und Art. 77 AsylG).

Die Notwendigkeit, Aussen- und Binnenperspektive zu verbinden, rieb sich aber in der Vergangenheit häufig an innenpolitischen Auseinandersetzungen. Ein Blick auf die verschiedenen, von breiten Teilen der Bevölkerung unterstützten Abstimmungen gegen die Migrationspolitik in den 1990er Jahren machen deutlich, unter welchem Erfolgsdruck selbst eine holistische Perspektive operieren muss, insbesondere wenn es um Asyl- und irreguläre Migration geht. Im Folgenden richten wir unsere Aufmerksamkeit auf diese beiden Migrationsformen.

\section{Die Asylmigration}

Nach 1945 anerkannte die Schweiz ihre Verantwortung für die Wegweisung jüdischer Flüchtlinge an der Grenze während des Zweiten Weltkriegs ${ }^{16}$. Der Bundesrat unterstrich deshalb seinen Willen, die humanitäre Tradition aufrechtzuerhalten und unterzeichnete 1955 die Genfer Konvention, die den Flüchtlingsstatus seit 1951 völkerrechtlich regelte. In den kommenden zwei Jahrzehnten betrieb das Land eine liberale Asylpolitik und bot insbesondere Flüchtlingen aus

15 Damir Skenderovic und Gianni D'Amato, Mit dem Fremden politisieren. Rechtspopulistische Parteien und Migrationspolitik in der Schweiz seit den 1960er Jahren, Zürich, Chronos, 2008, S. 157.

16 Siehe Carl Ludwig, ,Die Flüchtlingspolitik der Schweiz in den Jahren 1933 bis 1955 : Bericht an den Bundesrat zuhanden der eidgenössischen Räte“, in Carl Ludwig, Die Flüchtlingspolitik der Schweiz seit 1933 bis zur Gegenwart: Beilage zum Bericht des Bundesrates (...), Bern, [s.n.], 1957, S. 1-376. 
kommunistischen Staaten grosszügige Aufnahme. Die damaligen Verfahren waren relativ einfach und in der Regel wurde den Flüchtlingen Asyl erteilt. So wurden 195614000 Ungarn aufgenommen und 196812000 Tschechoslowa$\operatorname{ken}^{17}$.

Diese Flüchtlinge waren häufig gut ausgebildet und erhielten - nicht zuletzt wegen dem konsensfähigen Antikommunismus - eine herzliche Aufnahme. Die Ankunft indessen von wenigen hundert chilenischen Flüchtlingen, die nach dem Putsch von Pinochet 1973 in der Schweiz Zuflucht suchten, führte zu einer ersten kontroversen Debatte über die Legitimität des ihnen damals verliehenen Flüchtlingsstatus. Zwischen 1979 und 1982 gewährte die Schweiz noch 8000 vietnamesischen und kambodschanischen Boat-People Schutz auf der Basis von jährlichen Kontingenten. Deren Integration erwies sich als schwieriger als jener der vorherigen Flüchtlingsgruppen ${ }^{18}$.

Im Jahr 1981 gab sich die Schweiz erstmals ein Asylgesetz, das die bislang grosszügige Praxis kodizifizierte. Es definierte die Regeln der Vergabe des Flüchtlingsstatus, bestimmte die Verfahren und gab dem Bund ein Instrument politischer Intervention in die Hand. Allerdings oblag die Verantwortung der Implementierung dieser Politiken den Kantonen. In Bereichen wie der Wohlfahrt, Weiterbildung und Repatriierung war die Gestaltungsfähigkeit der Kantone entscheidend, was unterschiedliche Praxen zwischen den Kantonen zur Folge hatte ${ }^{19}$.

Nach 1981 machten sich zwei neue Trends bemerkbar: Die Zahl der Gesuche, die sich während den 70er Jahren auf 1000 pro Jahr eingependelt hatten, stieg markant und exponentiell an. Ausserdem kam ein grosser Teil der Asyl Suchenden (mit Ausnahme der Polen 1982) aus dem „Süden“, mehrheitlich aus Regionen wie der Türkei, Sri Lanka, dem Mittleren Osten, Afrika oder Asien. Anders als bei den antikommunistischen Flüchtlingen verfügten diese Bewerber nicht immer über ausreichende berufliche Qualifikationen und Abschlüsse. Ab Mitte der 1980er Jahre wurde die Asylpolitik zu einem politisch sensiblen Thema, da die Wanderung aus Fluchtgründen vom humanitären Völkerrecht geschützt wird. In öffentlichen Debatten wurden die Schutz suchenden Menschen häufig abschätzig als Asylanten betitelt, um anzuzeigen, dass ihre Wanderungsmotive unlauter seien, dass sie nicht legitimiert seien, den Flüchtlingsstatus zu beanspruchen, da sie nur gekommen seien, um einen Aufenthalt zu erschleichen. Dieser in der Öffentlichkeit stark präsente Diskurs führte dazu, das Asylgesetz mittels Revisionen den damals vermeintlich politischen Notwendigkeiten anzupassen, die Verfahren dementsprechend enger auszulegen und weniger Gesuche

17 Denise Efionayi-Mäder et al., „Switzerland“, in Jan Niessen und Yongmi Schibel (Hg.), EU and US Approaches to the Management of Immigration: Comparative Perspectives, Brussels, Migration Policy Group, 2003, S. 491-519.

18 Lorena Parini und Matteo Gianni, La tension entre précarité et intégration: politique à l'égard des migrants en Suisse, Genève, Département de science politique de l'Université de Genève, 1996, 21 S. Lorena Parini und Matteo Gianni, ,Enjeux et modifications de la politique d'asile en Suisse de 1956 à nos jours“, in Hans Mahnig (Hg.), Histoire de la politique de migration, d'asile et d'intégration en Suisse depuis 1948, Zurich, Seismo, 2005, S. 189-252.

19 Thomas Holzer und Gerald Schneider, „Die Schattenseiten der dezentralen Durchführung von Asylverfahren: Erfahrungen aus der Schweiz, Perspektiven für die Europäische Union“, in Hans-Rudolf Wicker, Rosita Fibbi und Werner Haug (Hg.), Migration und die Schweiz: Ergebnisse des Nationalen Forschungsprogramms „,Migration und interkulturelle Beziehungen“, Zürich, Seismo, 2003, S. 161-179. 
anzuerkennen, selbst dann, wenn die Gesuchsteller aus Kriegsgebieten kamen. Einen groben Indikator für diesen Trend ergibt ein Blick auf die damaligen Anerkennungsquoten: Betrugen diese im Zeitraum zwischen 1975 und 1979 86 Prozent, sanken die Zahlen zwischen 1980 und 1984 auf 46 Prozent und zwischen 1985 und 1990 auf 6 Prozent ${ }^{20}$.

In der öffentlichen Debatte gewann das Asylthema immer mehr an Gewicht und verdrängte die einst umstrittene Frage der Arbeitsmigration vom politischen Parkett. Trotz geringerer Anerkennungsraten in den 1990er Jahren konnten viele Asyl Suchende aus humanitären Gründen in der Schweiz bleiben. Obschon die Kantone deren Rechte für die erste Phase ihres Aufenthalts meist restriktiv regelten - beschränkter Zugang zum Arbeitsmarkt und zu den Sozialleistungen, Verhinderung der Familienzusammenführung - konnten jene Bewerber, denen die Schutzwürdigkeit zugebilligt wurde, sich später dauerhaft niederlassen. In den 1990er Jahren brachten insbesondere die Balkankriege neue Flüchtlingswellen aus Bosnien und Kosovo in das Land. Einige dieser Kriegsflüchtlinge hatten Verwandte in der Schweiz, meist waren es Arbeitsmigranten, die noch vor dem Bürgerkrieg emigriert waren. Zwischen 1990 und 2002 wurden in der Schweiz 146587 Asylgesuche aus der kriegsgeplagten Balkanregion gestellt ${ }^{21}$.

In der Öffentlichkeit kam es in den 1990er Jahren wegen der steigenden Asylzahlen erneut zu Unmutsbekundungen, nicht zuletzt, weil die Arbeitslosigkeit auf Grund der bereits erwähnten Strukturkrise der Schweizer Wirtschaft in ungewohntem Masse stieg. Der Bundesrat leitete deshalb erneut administrative und rechtliche Massnahmen ein, um die Gesuchsverfahren zu beschleunigen und die getroffenen Entscheidungen effektiver umzusetzen. Das Asylgesetz blieb eine Baustelle und wurde 1999 erneut revidiert. Im neuen Gesetz wurden neue Gründe eingeführt, die Asyl Suchende von einem Zugang zum Verfahren abhalten konnten. Insbesondere Gesuchsteller, die sich illegal im Land aufhielten und keine Identitätspapiere vorweisen konnten, sollten keine Gelegenheit mehr erhalten, langfristig in der Schweiz zu bleiben. Als Konzession an humanitär formulierte Vorbehalte seitens der Hilfswerke regelte das neue Gesetz die zeitlich beschränkte kollektive Aufnahme von Kriegsflüchtlingen, was insbesondere von Kosovaren und Bosniaken genutzt wurde. Nach den Balkankriegen gingen schätzungsweise 60000 Personen wieder zurück in ihre Herkunftsgebiete, während ungefähr 10000 Kriegsflüchtlinge definitiv in der Schweiz blieben. Der Anteil jener, die sich illegal in der Schweiz durchschlagen, ist nicht bekannt ${ }^{22}$.

Obschon die politische Atmosphäre während den verschiedenen politischen Debatten um die Revision der Revision des Asylgesetzes während den 1980er und 1990er Jahren an die Überfremdungsdiskurse vergangener Ausländer-

20 Denise Efionayi-Mäder, ,Asylpolitik der Schweiz 1950-2000“, Asyl, Nr. 2, 2003, S. 3-9.

Allerdings nahm der Anteil ab der zweiten Hälfte der 90er Jahre wieder leicht zu (9 Prozent) und hielt sich bis 2004. Ab 2005 stieg die durchschnittliche Anerkennungsquote wieder deutlich auf 18 Prozent an (siehe <http://www.bfm.admin.ch/etc/medialib/data/migration/statistik/asylstatistik/uebersichten. Par.0003.File.tmp/Asyl-Uebersicht-Jahre-Total-d.pdf>).

21 Bülent Kaya, „Switzerland“, in Jan Niessen, Yongmi Schibel und Cressida Thompson (Hg.), Current Immigration Debates in Europe: A Publication of the European Migration Dialogue, Brussels, Migration Policy Group, 2005, S. 383-398.

22 Denise Efionayi, Josef Martin Niederberger und Philippe Wanner, ,,Switzerland Faces Common European Challenges“, Migration Information Source (MPI), Februar 2005. 
abstimmungen erinnerte, kann nicht übersehen werden, dass Asylbewerber auf dem Arbeitsmarkt ziemlich genau von jenen Branchen rekrutiert wurden, die zuvor Saisonarbeitskräfte beschäftigten. Obwohl ausdrücklich nicht als Arbeitsmigration verstanden, tragen Arbeitskräfte mit Asylstatus zur Wirtschaftsentwicklung bei. Etienne Piguet und Andreas Wimmer haben als erste die Hypothese geäussert, Asylbewerber seien die neuen "Gastarbeiter" der Schweiz"23. Jürg Kuster und Guido Cavelti haben in einer Forschungsstudie diese These bestätigt ${ }^{24}$. Indes, wenn Asylbewerberinnen und -bewerber in den gleichen Branchen wie die Saisonniers arbeiten, ist dies in grossem Mass den behördlichen Massnahmen geschuldet, die den Zugang zum Arbeitsmarkt regulieren. Da seit den 1990er Jahren Angehörige ausserhalb der EU keine Saisonbewilligungen mehr erhalten, die Nachfrage nach unqualifizierter Arbeit auf dem Arbeitsmarkt aber weiterhin gedeckt werden muss, konnten zur Not nur Asylbewerber aktiviert werden ${ }^{25}$. Diese erweisen sich für den peripheren Arbeitsmarkt als optimal flexibel einsetzbar. Insbesondere die Hotellerie und das Bau- und Reinigungswesen profitieren von dieser Flexibilität ${ }^{26}$. Diese Situation ist auf einen ausgetrockneten Arbeitsmarkt selbst für niedrigqualifizierte Arbeiten zurückzuführen. Trotz der behördlichen Ausrichtung auf die hochqualifizierte und europäische Migration findet in deren Schatten seit den 1990er Jahren eine Einwanderung statt, die sich ihren ökonomischen Platz behauptet, obschon sie auf einer symbolisch-diskursiven Ebene unerwünscht bleibt, da deren Motive weniger respektabel erscheinen (Flucht aus Elend und aus Gewaltregionen). Die symbolische Einordnung dieser der Not folgenden Migration hat Konsequenzen für die politische und soziale Einordnung unerwünschter Formen der Migration, insbesondere der irregulären Migration.

\section{Die irreguläre Migration}

Als Antwort auf die steigenden Asylzahlen der 1980er Jahre und aufgrund der allgemein verbreiteten Einstellung, die Mehrheit der Asyl Suchenden seien aufgrund falscher Angaben eingewandert, wurden im Jahrzehnt danach eine Reihe von Politiken entworfen, die das Ziel hatten, das Asylrecht restriktiver zu deuten, um so die Zahl der Asyl Suchenden zu senken und den aufstrebenden rechtspopulistischen Parteien, die das Asylthema beharrlich bewirtschafteten, den Wind aus den Segeln zu nehmen. So wurden zahlreiche Beschränkungen in der Sozialhilfe, im Zugang zum Arbeitsmarkt und in anderen Lebensbereichen (Bildung, medizinische Versorgung, Mobilität) eingeführt, die nur für Asyl Suchende gelten und diese in ihrer sozialen und räumlichen Mobilität massiv einschränken. Auf europäischer Eben wurde mit dem Schengener Abkommen

23 Etienne Piguet und Andreas Wimmer, „Les nouveaux „Gastarbeiter“? Les réfugiés sur le marché du travail suisse", Revue de l'intégration et de la migration internationale, Bd. 1, Nr. 2, 2002, S. 233-257.

24 Jürg Kuster und Guido Cavelti, „Rekrutierung ausländischer Arbeitskräfte: die Bedeutung ausländerund asylrechtlicher Bestimmungen“, in Hans-Rudolf Wicker, Rosita Fibbi und Werner Haug (Hg.), Migration und die Schweiz: Ergebnisse des Nationalen Forschungsprogramms „Migration und interkulturelle Beziehungen “, Zürich, Seismo, 2003, S. 259-274.

25 Rosita Fibbi und Janine Dahinden, „Les requérants d'asile et le travail: déclassés? indispensables? bienvenus ?", Asyl, Bd. 19, Nr. 3, 2004, S. 21.

26 Etienne Piguet, Stefano Losa und Jean-Hugues Ravel, „Demandeurs d'asile et travailleurs étrangers clandestins sur le marché du travail suisse“", Asyl, Bd. 17, Nr. 2, 2002, S. 3-8. 
von 1985 und der Konvention von Dublin von 1990 ein gesamteuropäisches Migrationsregime aufgebaut, das einerseits mit der Abschaffung der Grenzkontrollen einen gemeinsamen territorialen Raum für die EU-Bürger definierte, andererseits für Angehörige einer Reihe von Drittstaaten Visaerfordernisse einführt, die eine geordnete Einwanderung aus diesen Staaten trotz gegenteiliger Absicht unmöglich macht ${ }^{27}$. In der Schweizer Gesetzgebung sind diese Abkommen seit 2008 ebenfalls in Kraft. Mit der Nennung sicherer Drittstaaten, in die Asyl Suchende zurückgeschickt werden können, ohne dadurch das Non-Refoulement-Gebot der Genfer Konvention zu verletzen, wurde zunächst in den ehemaligen kommunistischen Staaten Mittel- und Osteuropas eine Pufferzone um die EU errichtet. Bei deren Integration in die Europäische Union ist diese weiter nach Osten und Süden verschoben worden. Um mehrfache Asylanträge zu unterbinden, sind seither Asylentscheidungen der Mitgliedstaaten für alle Unterzeichnerstaaten bindend. Demnach darf ein Asyl Suchender nur in einem Staat Asyl beantragen. Bilaterale Wiederaufnahmevereinbarungen ermöglichen eine Ausweisung von abgewiesenen Asyl Suchenden in sichere Drittstaaten, die diese zuvor durchquert haben. Diese Massnahmen haben, nebst der Einführung der Möglichkeit einer Beantragung des Asylgesuchs im Herkunftsland (,,incountry processing“) und der Bestrafung der Luftfahrtsunternehmen (,carrier sanctions") bei einem Transport von Passagieren, die keine Einreisegenehmigung haben, versucht, die Migrationsflüsse in eine andere Richtung zu kanalisieren. Ausserdem haben die EU-Staaten die Einwanderungskontrolle territorial ausgelagert und durch die Einführung von sogenannten "pre-boarding Checks“ auf die Luftfahrtsunternehmen übertragen, was einer Privatisierung der Grenzkontrollen gleichkommt (,remote control“).

Die kombinierte Wirkung dieser Regelungen hatte zur Folge, dass mit einer zunehmend restriktiv interpretierten Definition der Flüchtlingseigenschaft gemäss Artikel 1 der Genfer Konvention die Zahl der Asyl Suchenden in Westeuropa nach 1992 drastisch gesenkt werden konnte. Der anfängliche Erfolg der neuen Massnahmen schien jedoch gegen Ende der 1990er Jahre wieder zu verpuffen. Der erneute Anstieg der Asyl Suchenden lässt sich damit erklären, dass mit der Schliessung des „Asylkanals“ viele Migranten und Asyl Suchende auf neue, irreguläre Pfade des Grenzübertritts ausgewichen sind, um ihr Auswanderungsprojekt realisieren zu können, so wie zehn Jahre zuvor die Kündigung der Gastarbeiterprogramme viele Ausreisewillige auf den „Asylweg“ umgeleitet hatte. Es gibt ausserdem etliche Hinweise dafür, dass diese Möglichkeit vermehrt von Menschenschmugglern monopolisiert wird, um Migrantinnen und Migranten illegal einreisen zu lassen ${ }^{28}$. Doch was bedeutet illegale Migration?

Illegale Migration bezeichnet in diesem Sinn sämtliche Phänomene in Verbindung mit der rechtswidrigen Einwanderung und dem unerlaubten Aufenthalt von ausländischen Staatsangehörigen in Nationalstaaten. Andere Bezeichnungen für diese Situation heissen „klandestine Migration“, „,undokumentierte Migration“ oder ,,irreguläre Migration“. Die Vielzahl der Beschreibungen mag

27 Sandra Lavenex und Emek M. Uçarer (Hg.), Migration and the Externalities of European Integration, Lanham, Lexington Books, 2002.

28 Khalid Koser, „The Smuggling of Asylum Seekers into Western Europe: Contradictions, Conundrums, and Dilemmas", in David Kyle und Rey Koslowski (Hg.), Global Human Smuggling: Comparative Perspectives, Baltimore, MD, Johns Hopkins University Press, 2001. 
daran erinnern, dass die Irregularität der Migration eine Frage der Perspektive ist $^{29}$. Zu ihren Erscheinungsformen zählen:

$\checkmark$ der irreguläre (illegale oder getäuschte) Übertritt und der illegale Aufenthalt (klassische Sans-Papier Situation)

$\checkmark$ der irreguläre Übertritt und der legale Aufenthalt (z.B. Asyl Suchende)

$\checkmark$ der legale Übertritt und die Übertretung der Aufenthaltsdauer, die in eine illegale Aufenthaltssituation mündet oder die Visabestimmungen verletzt (z.B. sogenannte „Overstayers“"30.

Der Begriff der „Sans-Papiers“ für Personen ohne gültige Aufenthaltspapiere wurde Anfang der 1970er-Jahre in Frankreich geprägt, um stigmatisierende Bezeichnungen, die mit Delinquenz konnotiert werden (Illegale, clandestins) zu ersetzen. Die englischsprachige Fachliteratur unterscheidet vielfach zwischen Papierlosen (undocumented migrants), die illegal ins Land gelangt sind und ein Aufenthaltsrecht besassen, und legal eingereisten Einwanderern, die ihre Aufenthaltsberechtigung verloren haben (overstayers). Dies kann beispielsweise infolge der Nichterneuerung der Aufenthaltsbewilligung nach Ablauf eines Touristenvisums, Abschluss eines Studiums, Ablehnung eines Asylgesuchs sowie durch Verlust eines Arbeitsplatzes, Scheidung, Tod des Ehepartners oder wegen Fürsorgeabhängigkeit, Straffälligkeit sowie am Ende eines legalen Kurzaufenthalts geschehen.

Der rechtliche Situation von Personen ohne Aufenthaltsstatus ist ausgesprochen komplex, da zwar Menschenrechte sowie gewisse sozial- und arbeitsrechtliche Ansprüche für diese Gruppe ebenso Geltung haben wie für registrierte Migranten, von den Betroffenen nur, wenn überhaupt, mit dem Risiko einer Ausweisung eingefordert werden können. Obwohl ein illegaler Aufenthalt generell als selbstverschuldet betrachtet wird, ist nicht $\mathrm{zu}$ übersehen, dass zahlreiche Betriebe und Privathaushalte von der verfügbaren, meist billigen Arbeitskraft der Papierlosen profitieren. Ferner haben die vermehrten Zulassungsbeschränkungen im Ausländer- und Asylbereich zweifellos zu einer Zunahme der Illegalität beigetragen. Die Ursachen illegaler Migration sind aber ebenso vielschichtig, wie die Lebenslagen und Laufbahnen von Personen ohne Aufenthaltsstatus vielfältig sind. Erfahrungsgemäss lassen sich deshalb illegale Migrationsbewegungen selbst bei Bestehen legaler Einwanderungsperspektiven nicht vermeiden. Gewiss ist nur, dass erst die Personenfreizügigkeit das Phänomen ganz zu überwinden vermag.

Auch wenn wenige Informationen über Papierlose bestehen, erlauben Auskünfte von Fachpersonen und bruchstückhafte Daten Indikationen auf das Profil der betreffenden Personen ${ }^{31}$. In der Schweiz stammen sie mehrheitlich aus Lateinamerika (insbesondere die Frauen), aus den Nachfolgestaaten des ehemaligen Jugoslawiens, aus Osteuropa, der Türkei und vereinzelt aus afrikanischen und asiatischen Ländern. Ihr Alter liegt mehrheitlich zwischen 20 und 40 Jahren.

29 Bimal Ghosh, Huddled Masses and Uncertain Shores: Insights into Irregular Migration, The Hague, Martinus Nijhoff Publishers, 1998, S. 1.

30 Vgl. D'Amato, Gianni, Brigitta Gerber und Martina Kamm, Menschenschmuggel und irreguläre Migration in der Schweiz, Neuchâtel, Swiss Forum for Migration and Population Studies, 2005.

31 Denise Efionayi-Mäder und Christin Achermann, „Ohne Papiere, aber nicht rechtlos“, Soziale Sicherheit, Nr. 6, 2003, S. 360. 
Frauen sind besonders in grossen Städten in der Überzahl, doch halten sich auch Familien und alleinstehende Männer ohne Aufenthaltsberechtigung in der Schweiz auf. Papierlose sind relativ gut ausgebildet, deren Löhne fallen hingegen häufig geringer aus als bei den legalen Arbeitskräften. Die grosse Mehrheit von ihnen ist erwerbstätig, insbesondere in der Hausarbeit (Reinigung, Kinderbetreuung, Altenpflege etc.), Reinigungsbranche, Gastgewerbe, Baugewerbe, Landwirtschaft und im Sexgewerbe.

Bis Ende der 1990er Jahre blieb Illegalität im deutschsprachigen Raum ein Randthema, das mit einem Tabu belegt war und nur von anwaltschaftlichen Vertretern offen debattiert wurde. Forschungen haben indes geholfen, die Erkenntnis durchzusetzen, dass Zuwanderung in einer immer stärker vernetzten Welt durch nationale oder überstaatliche Regelungen nur beschränkt gesteuert werden kann. Im Spannungsfeld zwischen nationalstaatlicher Abgrenzung und sozioökonomischer Realität müssen Behörden und Nichtregierungsorganisationen bereits heute Lösungen im Umgang mit Sans-Papiers finden, die mit einer streng legal ausgelegten Praxis manchmal schwer zu vereinbaren sind. Die Forderung nach einer Politikgestaltung bleibt im Raum, die sowohl menschenrechtlich vertretbar als auch migrationspolitisch glaubwürdige Handlungsansätze entwickelt.

\section{Schlussbemerkungen}

Die Migrationsentwicklungen der letzten Jahre, mit denen sich die Schweiz wie andere westliche Staaten konfrontiert sieht, lassen sich deshalb nur ungenügend mit einem sektoriellen, auf einen Aspekt der Politik ausgerichteten Ansatz erfassen. Sicherlich sticht die ungleich „bessere“ Behandlung der „südeuropäischen“" Migrantinnen und Migranten, die bereits in den 1950er und 1960er Jahren in die Schweiz kamen, im Vergleich zu den Neuankömmlingen der 1980er und 1990er Jahre ins Auge. Die „Privilegierung“ der europäischen Migration ist zum grossen Teil auf die damalige starke Nachfrage von Arbeitskräften zurückzuführen, die bilateral mit Auswanderungsstaaten geregelt wurde. Selbst unter diesem Regelmechanismus kam es zwar zu politischen Verwerfungen, wie ein Blick auf die permanent gegen die „Überfremdung“ politisierenden populistischen Bewegungen von damals zeigt. Diese konnten allerdings vom Bundesrat, wenn auch mit Mühe, politisch ausgeglichen werden, da es grundsätzlich um eine erwünschte Arbeitsmigration ging ${ }^{32}$. Im Zuge der Zuwanderung von Flüchtlingen aus dem Süden ab Mitte der 1980er Jahre, der kriegerischen Entwicklungen in Südosteuropa in den 1990er Jahren und der zunehmend als drängend perzipierten Wichtigkeit der irregulären Migration kam es zu einem Wendepunkt in der Politik, welcher die klare sektoriellen Aufteilung der Migrationspolitik aufweichte: Einerseits wurden die Territorien des ehemaligen Jugoslawiens anfangs der 1990er Jahre für die Rekrutierung gesperrt, andererseits wurde die Notwendigkeit seitens des Bundesrates unterstrichen, künftig die Migrationspolitik umfassend anzugehen ${ }^{33}$. Seither haben sich die Berührungspunkte zwischen

32 Damir Skenderovic und Gianni D'Amato, op. cit.

33 Peter Arbenz, Bericht über eine Migrationspolitik: Entwurf vom 25.3.1994, Bern, EJPD, 1994. Peter Arbenz, Bericht über eine schweizerische Migrationspolitik, s.1., s.n., 1995. Peter Arbenz, ,Von der Asylpolitik zu einer Migrationspolitik“, in Philippe Henry, Gaston Gaudard und Peter Arbenz (Hg.), 
der Arbeitsmarkt-, Asyl- und Entwicklungspolitik stetig verstärkt. Dies ist insbesondere ersichtlich aus der Koppelung der Rückkehrpolitik mit den Strukturhilfen in den ehemaligen Herkunftsgebieten und dem Engagement des Bundesrates, präventiv gegen die irreguläre Migration vorzugehen ${ }^{34}$.

Vermehrt kamen in den letzten Jahren die Bestrebungen der Schweizer Regierung wie auch der anderer westlicher Staaten zum Tragen, in Partnerschaften mit Regierungsmitgliedern, Politikern, Angehörigen der Bürgergesellschaft und Wissenschaftlern aus dem Süden in entsprechenden internationalen Foren die anstehenden Probleme gemeinsam anzugehen ${ }^{35}$. Doch auch in dieser Politik setzte sich der dominierende Diskurs durch, wonach Migration im Grunde genommen ein Problem darstellt, das über angemessene Politiken „behoben“ und geregelt werden müsste. Während die repressive Politikvariante mit Grenzkontrollen und Wahrung der politisch-territorialen Souveränität argumentiert, möchte die liberale Seite die Ursachen der Migration anpacken (meist Armut und Gewalt), damit Menschen nicht mehr auswandern müssen. Migration müsste demnach als ein dysfunktionaler Prozess unterbunden werden, da beide Seiten Migration als eine ungewollte Notwendigkeit betrachten. Hier lässt sich ein roter Faden von den ehemaligen Kolonialbehörden zu einigen der gegenwärtigen Agenturen der Entwicklungszusammenarbeit ziehen, welche Wanderarbeiter und Wanderarbeiterinnen schon immer am liebsten zu Hause gesehen hätten. $\mathrm{Da}$ allerdings die reichen Industrieländer nach wie vor ausländische Arbeitskräfte benötigen, münden die bei diesen Anlässen propagierten Empfehlungen zwar nicht in ein vollständiges Verbot der Migration von wenig qualifizierten Arbeiterinnen und Arbeitern, sondern häufig in die Propagierung der Idee, wonach gerade die ,zirkuläre Migration“ zu einer beidseits erwünschten ,winwin-Situation" führen könnte, sowohl für die Herkunfts- und Destinationsländer als auch für die Migranten und Migrantinnen von Nutzen wäre. Hierzu bleibt einzuwenden, dass gemäss dieser Vorstellung am Ende des ,Zirkulationsprozesses" die Migranten und Migrantinnen wieder zu Hause sind ${ }^{36}$.

Sicherlich ist Migration ein Aspekt der Moderne, der in den gegenwärtigen Analysen zu wenig mit den tiefgreifenden Transformationsprozessen unserer Epoche gekoppelt wird. Gerade aber aus dieser Situation gesellschaftsübergreifenden Wandels heraus erklärt sich die Komplexität, Verwobenheit, Variabilität und Kontextualität eines Mehrebenen-Phänomens, wie der Migration, die ihren Ursprung in der strukturell ungleichen Verteilung des Wohlstandes in der Weltgesellschaft hat. Allerdings ist mit diesem strukturellen Argument nicht gesagt, dass eine Welt ohne grösseres Wohlstandsgefälle eo ipso auch zu weniger Migration führen würde. Es ist eher wahrscheinlich, dass es zu einer anderen Art der Migration käme. Ökonomische Faktoren erklären deshalb nur einen Teil des Phänomens, sie reichen aber allein nicht aus, um die komplexe Einbettung des Migrationsphänomens angesichts des tiefen Wandels unserer globalen Zivilisation zu erfassen.

Die Schweiz als Asylland, Biel, Ed. Libertas Schweiz, 1995, S. 65-81. Bundesamt für Wirtschaft und Arbeit, Bericht über Konzeption und Prioritäten der schweizerischen Ausländerpolitik der neunziger Jahre, Bern, Eidg. Drucksachen- und Materialzentrale, 1991.

34 Projektteam PiM, Abschlussbericht Prävention irregulärer Migration, Bern, [s.n.], 2003.

35 Siehe den Beitrag von Pietro Mona (EDA) in diesem Dossier.

36 Stephen Castles, „Understanding Global Migration: A Social Transformation Perspective“, Paper for the IMISCOE Conference on Theories of Migration and Social Change, St Anne's College, University of Oxford, 1-3 July 2008. 
Wollten wir diesen Wandel verstehen, so müssten wir ein Verständnis haben, welches von westlichen postindustriellen Gesellschaften ausgeht und geprägt ist von global wirksamen Restrukturierungen sowohl auf der Ebene der Investitionen als auch auf der der Produktion und des Handels von Gütern und Wissen. In den entwickelten Ländern kam es insbesondere nach der Krise von 1973 zur Schliessung der alten Industrien, zur Restrukturierung des Arbeitsmarktes, der Erosion des Wohlfahrtsstaates und der Auflösung ehemals organisierter proletarischer Milieus. Diese Restrukturierungsprozesse haben zu einer Realität geführt, welche die Migration von hoch- als auch von unqualifizierten Arbeitskräften fördert. In weniger entwickelten Ländern hat diese aus der nördlichen Hemisphäre stammende globale Transformation zu Rationalisierung und Zerstörung ländlicher Lebensweisen, Erosion lokaler Sozialordnungen, Landflucht und Entstehung von Barackensiedlungen in den Grossstädten geführt, welche allesamt die Migration ankurbeln.

Schlussendlich sind diese beobachteten Prozesse auf eine neue Segmentierung der internationalen Arbeitsmärkte zurückzuführen. Den gesicherten Arbeitsstellen in den zentralen Bereichen gegenwärtiger Produktionsweisen stehen die flexiblen Jobs in den peripheren Arbeitsmärkten gegenüber. Die Möglichkeit, in den privilegierten Märkten eine entsprechende Arbeitsstelle zu finden, hängt nicht allein vom Humankapital ab, also von Wissen und Kompetenzen, sondern auch vom Geschlecht, der Herkunft und dem legalen Status. Jede einzelne Migrationsgeschichte widerspiegelt infolgedessen den komplexen Zusammenhang zwischen dem Wandel der Arbeitsmärkte und den grossen (post-)industriellen Transformationsprozessen im Süden und im Norden der Welt.

Eine Politik, die Migrationsphänomene produktiv angehen will, muss ihre Handlungsperspektiven auf das Wissen stützen, dass Migration erstens zur Menschheitsgeschichte gehört und dass zweitens die gegenwärtige Transformationsphase, die alle Teile des Globus gleichzeitig, wenn auch nicht gleich stark trifft, einen neuen Migrationsmodus geschaffen hat. Wer diese Prozesse beeinflussen will, braucht einen entsprechend breiten Deutungsrahmen des Phänomens, um es später realistisch deklinieren zu können.

Eine solche Gesamttheorie der Migration, auf das hat Stephen Castles mehrfach hingewiesen, gibt es zur Zeit nicht, ebenso wenig wie es eine globale Migrationspolitik gibt, im Sinne eines Code of Conduct etwa, der von einer Mehrzahl der Staaten geteilt würde. $\mathrm{Zu}$ disparat sind die Interessen der Regierungen, als dass sie sich auf ein gemeinsames Verständnis des Migrationsmanagements einigen würden. Lediglich die Europäische Union hat auf regionaler Ebene einen solchen Konsens zustande gebracht. Der Preis dafür ist allerdings, dass die interne Migration als gewollte, hochqualifizierte Mobilität fungiert, während die Bewegung von wenig qualifizierten Migranten und Migrantinnen, die von ausserhalb der EU kommen, als ungewollte Migration designiert worden ist. Mobilität wird so zum erwünschten Gut, weil sie dem Selbstverständnis offener, selbstbestimmter Gesellschaften entspricht, während Migration den Obolus des Zwangs mit sich führt, der gemeinhin mit archaischen Gemeinschaften in Verbindung gebracht wird. In einem solch umstrittenen Deutungskontext kann Migrationsforschung nicht umhin, sich über die Bedürfnisse der Tagespolitik hinaus sich reflexiv mit den verschiedenen, teils widersprüchlichen Aspekten der Moderne auseinanderzusetzen. Gerade hierin liegt die Chance ihrer (Selbst)Aufklärung. 


\section{Bibliographie}

Arbenz, Peter, Bericht über eine Migrationspolitik: Entwurf vom 25.3.1994, Bern, EJPD, 1994.

Arbenz, Peter, Bericht über eine schweizerische Migrationspolitik, [s.1.], [s.n.], 1995.

Arbenz, Peter, „Von der Asylpolitik zu einer Migrationspolitik”, in Philippe Henry, Gaston Gaudard und Peter Arbenz (Hg.), Die Schweiz als Asylland, Biel, Ed. Libertas Schweiz, 1995, S. 65-81.

Bolzman, Claudio, Rosita Fibbi und Marie Vial, Secondas - secondos: le processus d'intégration des jeunes adultes issus de la migration espagnole et italienne en Suisse, Zurich, Seismo, 2003.

Bundesamt für Wirtschaft und Arbeit, Bericht über Konzeption und Prioritäten der schweizerischen Ausländerpolitik der neunziger Jahre, Bern, Eidg. Drucksachen- und Materialzentrale, 1991.

Busset, Thomas, „La politique du refuge en Suisse 1820-1870. Réalité et mythe“, Zeitschrift des Schweizerischen Bundesarchivs, Nr. 25, 1999, S. 29-64.

Carigiet, Erwin, Ueli Mäder und Jean-Michel Bonvin (Hg.), Wörterbuch der Sozialpolitik, Zürich, Rotpunktverlag, 2003.

Castles, Stephen, „Understanding Global Migration: A Social Transformation Perspective“, paper for the IMISCOE Conference on Theories of Migration and Social Change, St Anne's College, University of Oxford, 1-3 July 2008.

Cerutti, Mauro, „L'immigration italienne en Suisse dans le contexte de la Guerre froide“, in Jean Batou, Mauro Cerutti und Charles Heimberg (Hg.), Pour une histoire des gens sans histoire: ouvriers, exclus et rebelles en Suisse: XIX ${ }^{e}-X X^{e}$ siècles, Lausanne, Editions d'En Bas, 1995, S. 213-231.

D’Amato, Gianni und Brigitta Gerber (Hg.), Herausforderung Integration: städtische Migrationspolitik in der Schweiz und in Europa, Zürich, Seismo, 2005.

Davis, Kingsley, "Social Science Approaches to International Migration", Population and Development Review, Bd. 14, Nr. 4, 1988, S. 245-261.

Efionayi, Denise, Josef Martin Niederberger und Philippe Wanner, „Switzerland Faces Common European Challenges“, Migration Information Source (MPI), February 2005.

Efionayi-Mäder, Denise, ,Asylpolitik der Schweiz 1950-2000“, Asyl, Nr. 2, 2003, S. 3-9.

Efionayi-Mäder, Denise et al., „Switzerland“, in Jan Niessen und Yongmi Schibel (Hg.), EU and US Approaches to the Management of Immigration: Comparative Perspectives, Brussels, Migration Policy Group, 2003, S. 491-519.

Efionayi-Mäder, Denise und Christin Achermann, „Ohne Papiere, aber nicht rechtlos“, Soziale Sicherheit, Nr. 6, 2003, S. 359-362.

Fibbi, Rosita und Janine Dahinden, „Les requérants d'asile et le travail: déclassés? indispensables ? bienvenus ?", Asyl, Bd. 19, Nr. 3, 2004, S. 21-27.

Fibbi, Rosita, Mathias Lerch und Philippe Wanner, L'intégration des populations issues de l'immigration en Suisse: personnes naturalisés et deuxième génération, Neuchâtel, Office fédéral de la statistique, 2005.

Freiburghaus, Dieter und Brigitte Guggisberg, „Die schweizerische Ausländerpolitik seit 1850: eine Analyse auf dem Hintergrund politisch-ökonomischer Paradigmen“, in Thomas Geiser, Hans Schmid und Emil Walter-Busch (Hg.), Arbeit in der Schweiz des 20. Jahrhunderts: wirtschaftliche, rechtliche und soziale Perspektiven, Bern, P. Haupt, 1998, S. 137-185.

Ghosh, Bimal, Huddled Masses and Uncertain Shores: Insights into Irregular Migration, The Hague, Martinus Nijhoff Publishers, 1998.

Holmes, Madelyn, Forgotten Migrants : Foreign Workers in Switzerland before World War I, Rutherford, Fairleigh Dickinson University Press, 1998.

Holzer, Thomas und Gerald Schneider, „Die Schattenseiten der dezentralen Durchführung von Asylverfahren: Erfahrungen aus der Schweiz, Perspektiven für die Europäische Union“, in Hans-Rudolf Wicker, Rosita Fibbi und Werner Haug (Hg.), Migration und die Schweiz: Ergebnisse des Nationalen Forschungsprogramms „Migration und interkulturelle Beziehungen“, Zürich, Seismo, 2003, S. 161-179.

Kaya, Bülent, „Switzerland“, in Jan Niessen, Yongmi Schibel und Cressida Thompson (Hg.), Current Immigration Debates in Europe: A Publication of the European Migration Dialogue, Brussels, Migration Policy Group, 2005, S. 383-398.

Koser, Khalid, „The Smuggling of Asylum Seekers into Western Europe: Contradictions, Conundrums, and Dilemmas“, in David Kyle und Rey Koslowski (Hg.), Global Human Smuggling: Comparative Perspectives, Baltimore, MD, Johns Hopkins University Press, 2001.

Kuster, Jürg und Guido Cavelti, „Rekrutierung ausländischer Arbeitskräfte: die Bedeutung ausländerund asylrechtlicher Bestimmungen“, in Hans-Rudolf Wicker, Rosita Fibbi und Werner Haug (Hg.), Migration und die Schweiz: Ergebnisse des Nationalen Forschungsprogramms „Migration und interkulturelle Beziehungen“, Zürich, Seismo, 2003, S. 259-274. 
Lavenex, Sandra und Emek M. Uçarer (Hg.), Migration and the Externalities of European Integration, Lanham, Lexington Books, 2002.

Ludwig, Carl, „Die Flüchtlingspolitik der Schweiz in den Jahren 1933 bis 1955 : Bericht an den Bundesrat zuhanden der eidgenössischen Räte“, in Carl Ludwig, Die Flüchtlingspolitik der Schweiz seit 1933 bis zur Gegenwart: Beilage zum Bericht des Bundesrates (...), Bern, [s.n.], 1957, S. 1-376.

Mahnig, Hans und Etienne Piguet, „Die Immigrationspolitik der Schweiz von 1948 bis 1998: Entwicklung und Auswirkungen“, in Hans-Rudolf Wicker, Rosita Fibbi und Werner Haug (Hg.), Migration und die Schweiz: Ergebnisse des Nationalen Forschungsprogramms „Migration und interkulturelle Beziehungen“" Zürich, Seismo, 2003, S. 65-108.

Parini, Lorena und Matteo Gianni, La tension entre précarité et intégration: politique à l'égard des migrants en Suisse, Genève, Département de science politique de l'Université de Genève, 1996, $21 \mathrm{~S}$.

Parini, Lorena und Matteo Gianni, „Enjeux et modifications de la politique d'asile en Suisse de 1956 à nos jours“, in Hans Mahnig (Hg.), Histoire de la politique de migration, d'asile et d'intégration en Suisse depuis 1948, Zurich, Seismo, 2005, S. 189-252.

Piguet, Etienne, Einwanderungsland Schweiz: fünf Jahrzehnte halb geöffnete Grenzen, Bern, Haupt Verlag, 2006.

Piguet, Etienne, Stefano Losa und Jean-Hugues Ravel, „Demandeurs d'asile et travailleurs étrangers clandestins sur le marché du travail suisse“, Asyl, Bd. 17, Nr. 2, 2002, S. 3-8.

Piguet, Etienne und Andreas Wimmer, „Les nouveaux „Gastarbeiter“? Les réfugiés sur le marché du travail suisse“, Revue de l'intégration et de la migration internationale, Bd. 1, Nr. 2, 2002, S. 233-257.

Projektteam PiM, Abschlussbericht Prävention irregulärer Migration, Bern, [s.n.], 2003.

Skenderovic, Damir und Gianni D’Amato, Mit dem Fremden politisieren. Rechtspopulistische Parteien und Migrationspolitik in der Schweiz seit den 1960er Jahren, Zürich, Chronos, 2008.

Urner, Klaus, Die Deutschen in der Schweiz: von den Anfängen der Kolonienbildung bis zum Ausbruch des Ersten Weltkrieges, Frauenfeld, Huber, 1976.

Vuilleumier, Marc, „L'emigrazione italiana in Svizzera e gli avvenimenti del 1898“, in AA. VV., Anna Kuliscioff e l'età del riformismo: atti del convegno di Milano, dicembre 1976, Roma, Mondo operaio, 1978, S. 85-103.

Vuilleumier, Marc, „Le mouvement ouvrier et les travailleurs italiens en Suisse jusqu'à la Première Guerre mondiale", Revue syndicale suisse, Bd. 82, Nr. 4, 1990, S. 102-116.

Wanner, Philippe, Migration und Integration: Ausländerinnen und Ausländer in der Schweiz, Neuchâtel, Bundesamt für Statistik, 2004. 